\title{
Spin-Sensitive Conductance in Magnetically Contacted Graphene Flakes
}

\begin{abstract}
S. KROMPIEWSKI
Institute of Molecular Physics, Polish Academy of Sciences, M. Smoluchowskiego 17, 60-179 Poznań, Poland

The aim of this study is to gain a deeper insight into the impact of geometrical dimensions (aspect ratio) and current direction (zigzag vs. armchair) on transport characteristics. It is found that there is a pronounced dependence of the giant magnetoresistance (in setups with 2 ferromagnetic electrodes), as well as the spin polarization of current (in the case of one paramagnetic and one ferromagnetic electrode) on the bias and gate voltages, meaning a possible electrical control of magnetic properties of these quantities.
\end{abstract}

PACS numbers: 72.25.Mk, 81.05.ue, 73.20.-r

\section{Introduction}

Carbon-based nanostructures are promising systems for future electronics, which may possibly replace conventional (Si-based) electronics. These systems may also be attractive for spintronics [1,2] if: (i) at least one of external electrodes is ferromagnetic, (ii) a back gate is ferromagnetic, or (iii) there are magnetic impurities. Hypothetically, (iv) finite graphitic structures might be spontaneously magnetic due to zigzag edges. Although the latter possibility is widely accepted theoretically, so far it has never been confirmed experimentally. This study deals with the scenario (i), generalizing previous studies [3] by modifying parametrization-dependent interface transparency and including asymmetric paramagnet/Gr/ferromagnet setups (with Gr standing for graphene).

\section{Method}

The computational method is based on a standard single-band tight-binding model for non-interacting electrons with hopping integrals $t_{\mathrm{G}}=2.7 \mathrm{eV}, t_{\mathrm{M}}$ and $t_{\mathrm{c}}$ for $\mathrm{Gr}$, metal electrode and interface, respectively. The on-site energies in $\mathrm{Gr}$ are proportional to the gate voltage (zero in the charge neutrality point), and they are spin-dependent in metallic electrodes. In particular, the paramagnetic electrode atomic potential is chosen so as to guarantee a half-band filling, whereas the bands of the ferromagnetic electrodes are Stoner split in such a way that the spin polarization is $50 \%$ (for more details on modelling and Green's function recursive method refer to Refs. $[3,4])$.

\section{Results and discussion}

Figure 1 shows the giant magnetoresistance (GMR) results for $c a .8 \mathrm{~nm}$ wide $\mathrm{Gr}$ nanoribbons with aspect ratio $A=$ width/length $=0.36$ and $A=3.4$ (ca. 10 times shorter ribbon). As expected, armchair (ac) edge nanoribbons reveal quasi-periodic behavior due to linear dispersion law at small energies. For the zigzag case, the energy dispersion law in the vicinity of the Dirac point is nonlinear due to low-energy edge states, so the peaks (dips) in the corresponding plots are no longer equidistant and there is no quasi-periodicity.

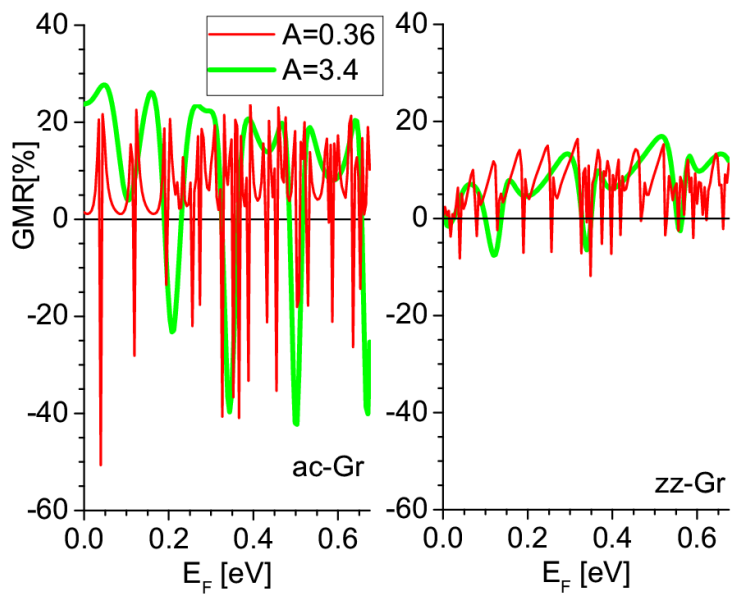

Fig. 1. GMR vs. energy in ferromagnetically contacted graphene for armchair-edge and zigzag-edge ribbons (left and right parts, respectively). The hopping parameters: $t_{\mathrm{M}}=2 t_{\mathrm{G}}, t_{\mathrm{C}}=0.4 t_{\mathrm{G}}$ and $t_{\mathrm{G}}=2.7 \mathrm{eV}$.

It should be noted that if only one of the electrodes is ferromagnetic and the system in between is intrinsically non-magnetic then there is no GMR. In that case a reorientation of the ferromagnetic electrode magnetization has no effect on the charge current. Then, a quantity of interest here is the spin-polarization of conductance, which very much like GMR is both chirality and aspect ratio dependent. Figure 2 presents the results for the ac-Gr nanoribbon with $A=0.36$. A nice periodicity is visible at low energies (voltages). Remarkably, as a function of both bias and gate voltages the polarization is subject to major changes, including the changes in sign. In prac- 
tice, it means that it is possible to control the current spin-polarization in graphene nanoribbons by electrical means, similarly as in carbon nanotube quantum dots $[5,6]$.
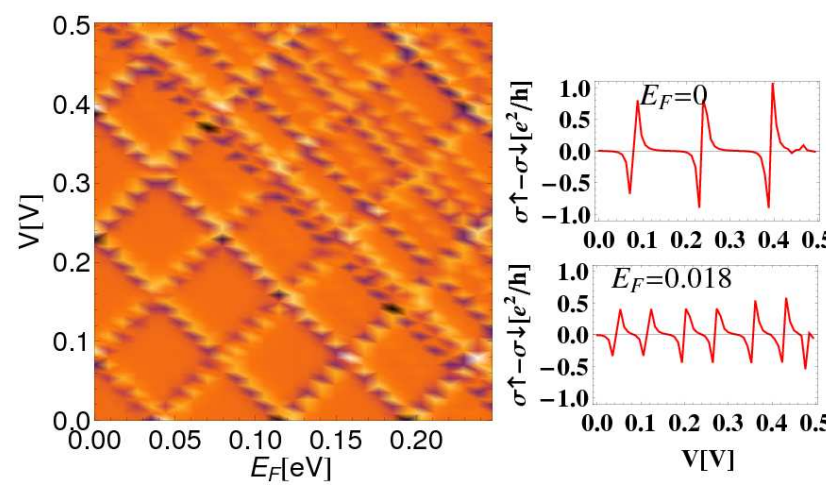

₹ $1.0 \quad E_{F}=0.018$

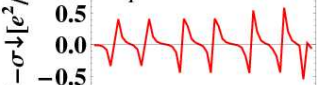

$\stackrel{1}{\leftarrow}-0.5$

$\begin{array}{lllllllllllllllll}0.0 & 0.1 & 0.2 & 0.3 & 0.4 & 0.5\end{array}$

$\mathrm{V}[\mathrm{V}]$

Fig. 2. Spin polarized conductivity in the paramagnet/ac-Gr/ferromagnet setup with $A=0.36$, and $t_{\mathrm{M}}=t_{\mathrm{C}}=t_{\mathrm{G}}$. The left part is a density plot, and the right one is the cross-section through $E_{\mathrm{F}}=0$ and $E_{\mathrm{F}}=0.018$.

In conclusion, it has been shown that Gr nanoribbons with ferromagnetic electrodes reveal pronounced GMR effects, clearly dependent on chirality and the aspect ra- tio. Noteworthy, the above mentioned observations hold also for the spin polarization of the conductance (current) if one external electrode is paramagnetic and the other - ferromagnetic. These findings show that both GMR and the current spin-polarization can be electrically controlled.

\section{Acknowledgments}

This work was partially supported by the Polish Ministry of Science and Higher Education as a research project No. N N202 199239.

\section{References}

[1] K. Tsukagoshi, B.W. Alphenaar, H. Ago, Nature (London) 401, 572 (1999).

[2] E. Hill, A.K. Geim, K. Novoselov, F. Schedin, P. Blake, IEEE Trans. Magn. 42, 2694 (2006).

[3] S. Krompiewski, Phys. Rev. B 80, 075433 (2009).

[4] S. Krompiewski, Physica Status Solidi B 242, 226 (2005).

[5] Ch.A. Merchant, N. Markovic, Phys. Rev. Lett. 100, 156601 (2008).

[6] I. Weymann, J. Barnaś, S. Krompiewski, Phys. Rev. B 78, 035422 (2008). 